\title{
Erratum to: Emulsification of Simulated Gastric Fluids Protects Wheat $\alpha$-Amylase Inhibitor 0.19 Epitopes from Digestion
}

\author{
Marica Consolini • Michela Sega • Chiara Zanetti • \\ Marina Fusi • Roberto Chignola • Marco De Carli • \\ Corrado Rizzi • Gianni Zoccatelli
}

Published online: 9 July 2011

(C) Springer Science+Business Media, LLC 2011

\section{Erratum to: Food Anal. Methods}

\section{DOI 10.1007/s12161-011-9227-z}

The original version of this article unfortunately contained mistakes. On page 2, right column, the fourth line from the last: "milligrams" should be "micrograms". On page 3, left column, the eleventh line from the first: "milligrams" should be "micrograms".

Marica Consolini and Michela Sega share the first authorship.

The online version of the original article can be found at http://dx.doi. org/10.1007/s12161-011-9227-z.

M. Consolini $\cdot$ M. Sega $\cdot$ C. Zanetti $\cdot$ M. Fusi $\cdot$ R. Chignola $\cdot$

C. Rizzi $\cdot$ G. Zoccatelli $(\bowtie)$

Dipartimento di Biotecnologie, Università degli Studi di Verona,

Strada Le Grazie, 15-CV1,

37134 Verona, Italy

e-mail: gianni.zoccatelli@univr.it

M. De Carli

Dipartimento di Medicina Interna, Azienda Ospedaliera,

Universitaria Santa Maria della Misericordia,

Udine, Italy 